\title{
Exploring the Relations Between Regret, Self-agency, and the Tendency to Repair Using Experimental Methods and Structural Equation Modeling
}

\author{
Evelien Bossuyt ${ }^{\mathrm{a}}$, Agnes Moors ${ }^{\mathrm{a}}$, and Jan De Houwer ${ }^{\mathrm{a}}$ \\ ${ }^{a}$ Ghent University, Department of Experimental Clinical and Health Psychology, Henri \\ Dunantlaan 2, 9000 Gent, Belgium. Email: Evelien.Bossuyt@Ugent.be, \\ Agnes.Moors@Ugent.be, and Jan.DeHouwer@Ugent.be.
}

\section{Author Note}

Preparation of this paper was supported by a Ph.D. fellowship of the Scientific Research Foundation, Flanders (FWO), Belgium, awarded to Evelien Bossuyt, FWO Research Project G.0223.13 and Methusalem Grant BOF09/01M00209 of Ghent University.

Please address correspondence to Evelien Bossuyt, Ghent University, Faculty of Psychology and Educational Sciences, Department of Experimental Clinical and Health Psychology, Henri Dunantlaan 2, 9000 Ghent, Belgium. Email: Evelien.Bossuyt@Ugent.be. Telephone: 0032 (0)9 26491 05. Fax: 0032 (0)9 2646489 


\begin{abstract}
Previous studies suggest that feelings of regret are elicited by events appraised as goal incongruent and caused by the self and that they are characterized by a tendency to repair the event. Study 1 investigated whether the appraisal of self-agency increases the tendency to repair. Participants played a game in which goal-congruent and goal-incongruent events were caused by themselves (self-agency) or by a die (circumstances-agency). The tendency to repair was measured via behavior and self-reports. Self-agency increased feelings of regret but not the tendency to repair. Moreover, our data rejected the idea that regret is more than other negative feelings associated with the tendency to repair. Study 2 confirmed the findings of Study 1 using autobiographical recall. Both studies provide support for a relation between self-agency and feelings of regret, but not between self-agency and the tendency to repair nor between the tendency to repair and feelings of regret.
\end{abstract}




\section{Exploring the Relations Between Regret, Self-agency, and the Tendency to Repair Using Experimental Methods and Structural Equation Modeling.}

Imagine that you are a finalist in a television quiz and one crucial decision keeps you from winning a million dollars. The concept is simple: one choice, everything or nothing. The show host presents you with two identical boxes, one containing the money, the other containing nothing. After some hesitation, you choose one of the boxes. The host opens the box you did not choose and you see that it contains the money. You realize that you chose the wrong box.

In situations like this, people are typically haunted by counterfactual thoughts on how things could have been if they had chosen differently. Mental time travel, vivid simulations of alternative actions, and other reverie are considered to be characteristic for the emotion of regret (Landman, 1993). Researchers have argued that besides these cognitive characteristics, regret has particular motivational qualities: Regret is said to be associated with the desire to undo the adverse outcomes of one's decisions, with reparative action tendencies, and with goal persistence (Zeelenberg \& Pieters, 2004a, 2006, 2007; Zeelenberg, van Dijk, Manstead, \& van der Pligt, 1998, 2000). In this paper, we investigated the relations between causing a negative outcome oneself, wanting to repair that outcome, and feelings of regret. We examined whether negative outcomes that are caused by oneself are associated with a stronger tendency to repair the outcome than negative outcomes that are not caused by oneself. In addition, our studies shed a new light on the relation between regret and the tendency to repair.

In contemporary emotion theories, emotions are often defined as multicomponential phenomena, consisting of (a) a cognitive component or appraisal of the situation, (b) a motivational component or action tendency, (c) a somatic component or (neuro)physiological responses, (d) a motor component or expressive behavior, and (e) a feeling component or 
subjective experience (Frijda, Kuipers, \& ter Schure, 1989; Moors, 2009; Roseman, 2001;

Scherer, 2005). The feeling component is considered a reflection of the other components (appraisal, action tendencies, physiology, and/or behavior) in consciousness (de Rivera, 1977; Scherer, 2005; Sonnemans \& Frijda, 1994). Particular feelings (i.e., patterns in the continuous flow of componential changes that surface in awareness) are labeled with emotion words such as regret, anger, fear, disappointment, and joy (Moors, 2009; Scherer, 2009).

Previous studies have investigated the appraisal process that shapes the feeling called regret. Appraisal is a process in which stimuli are evaluated on a number of appraisal variables, such as goal relevance, goal congruence, expectancy, coping potential, future expectancy, agency (i.e., the cause of an event: self, other, circumstances), and fairness (Frijda, 1986; Lazarus, 1991; Roseman, Antoniou, \& Jose, 1996; Scherer, 1988). These studies typically reveal that regret, together with guilt and shame, is part of a class of feelings that reflect that a stimulus is appraised as goal incongruent and caused by the self (Shefrin \& Statman, 1985; Zeelenberg et al., 2000). Other negative feelings reflect that a stimulus is appraised as goal incongruent and caused by others (e.g., anger) or by circumstances (e.g., fear, sadness, and disappointment; Roseman et al., 1996; van Dijk, van der Pligt, \& Zeelenberg, 1999; van Dijk \& Zeelenberg, 2002; Zeelenberg, van Dijk, \& Manstead, 1998; Zeelenberg, van Dijk, van der Pligt et al., 1998). The appraisal of agency thus seems crucial to differentiate regret from other negative feelings. Other appraisals differentiate regret from guilt and shame. Research has suggested that shame is felt when a person appraises herself as bad or as having failed to live up to personal standards (Keltner \& Buswell, 1996; Tangney, Miller, Flicker, \& Barlow, 1996; Tracy \& Robins, 2006) whereas guilt is felt when a person appraises that she has caused harm to another person (Keltner \& Buswell, 1996; Zeelenberg \& Breugelmans, 2008). 
Researchers have argued that the feeling of regret also reflects the activation of a specific action tendency: the tendency to repair the goal-incongruent event. For instance, Zeelenberg, van Dijk, Manstead, and van der Pligt (1998, p. 224) wrote "we conclude that the experience of regret involves a focus on the self as a cause of the event, and on possibilities for undoing the regret by changing the unfavourable outcome or by improving future performance." Other negative feelings reflect the activation of other action tendencies, such as the tendency to attack for anger, to obtain safety for fear, to disappear from sight for shame, and to become passive and turn away from the goal-incongruent event for disappointment (Frijda, 1986; Lazarus, 1991; Roseman, Wiest, \& Swartz, 1994; Zeelenberg, van Dijk, Manstead et al., 1998). Repairing a goal-incongruent event can take different forms. Some authors suggest that regret is related to one particular type of repair tendency: The tendency to redo but correct the initial behavior that gave rise to the goal-incongruent event (Roseman, 2011). Other authors do not seem to impose such restrictions (e.g., Zeelenberg, van Dijk, Manstead et al., 1998). For instance, when burning your birthday cake in the oven, you can repair this by making a new cake but setting the alarm clock (i.e., redo but correct) or by a wider range of actions such as buying a cake in the bakery or making another dessert. In the first, more restricted proposal, the appraisal of self-agency is logically implied in the definition of the action tendency: In order to redo but correct a behavior, the initial behavior must have been performed by oneself. In the second proposal, the concepts of appraisal and action tendency can be separated, which allows investigating the influence of one on the other.

In sum, previous research suggests that an appraisal of self-agency and a tendency to repair discriminate the feeling of regret from various other negative feelings. These studies focused either on the relation between regret and appraisals or on the relation between regret and 
action tendencies, neglecting the relation among appraisals and action tendencies. The aim of the present research is to examine the relation between the appraisal of self-agency and the tendency to repair.

We start by listing three possible ways in which the appraisal of self-agency and the tendency to repair can be related. A first possibility is that there is a causal relation. Several appraisal theorists (e.g., Frijda et al., 1989; Scherer, 1994) suggest that appraisals cause action tendencies. Thus, it has been argued that an appraisal pattern of goal-incongruence plus selfagency may cause the tendency to repair. For instance, van Dijk and Zeelenberg (2002, p. 329) propose that the motivational differences between regret and disappointment may be produced by differences in the appraisals of agency and/or legitimacy (for a similar position, see Martinez, Zeelenberg, \& Rijsman, 2011, p. 963). In principle, however, it is also possible that the tendency to repair causes the appraisal of self-agency or that they exert a mutual causal influence on each other (Moors \& Scherer, 2013; Scherer, 2009).

A second possibility is that there is not a causal relation between the appraisal of selfagency and the tendency to repair but only a temporal co-occurrence (Parkinson, 1997). A reason for such a co-occurrence may be that the appraisal of self-agency co-occurs with another appraisal and that this other appraisal causes the tendency to repair. For instance, goalincongruent events caused by oneself typically may be more easy to cope with than goalincongruent events caused by others or by circumstances. In turn, high coping potential may lead to the tendency to repair the event.

A third possibility is that there is not an actual but only a semantic relation between the appraisal of self-agency and the tendency to repair (Parkinson, 1997). For instance, layman's 
theories about regret may assume a link between self-agency and the tendency to repair (culturally inherited or acquired otherwise) that does not reflect reality.

In a first study, we examined whether the appraisal of self-agency has a causal influence on the tendency to repair. Previous research has examined the influence of this appraisal on a variety of behaviors that could be interpreted as manifestations of the tendency to repair. A first set of studies have shown that an appraisal of self-agency encourages a person to seek information about an obtained outcome and possible alternatives (Reb \& Connolly, 2009; Shani \& Zeelenberg, 2007). Information seeking can serve the goal to repair a goal-incongruent outcome or the goal to prevent a repetition of goal-incongruent outcomes in the future. A second set of studies show that self-agency leads to behavioral persistence or the escalation of commitment in investments (Staw, 1976) and in gambling (Clark, Crooks, Clarke, Aitken, \& Dunn, 2012; Clark, Lawrence, Astley-Jones, \& Gray, 2009). Behavioral persistence can be considered a manifestation of the tendency to repair if it is guided by the idea that it can undo a loss. A third set of studies suggest that the appraisal of self-agency (or self-blame) increases prosocial behavior toward an unrelated third party (Freedman, Wallington, \& Bless, 1967; Ketelaar \& Au, 2003; D. T. Regan, Williams, \& Sparling, 1972, but see Cialdini, Darby, \& Vincent, 1973; Rawlings, 1968, and J. W. Regan, 1971, for failed replications, and Cunningham, Steinberg, \& Grev, 1980, for boundary conditions). Helping a third party can be seen as an expression of the tendency to repair one's image rather than the goal-incongruent situation. There are also studies that measured the tendency to reconcile with or help the person that one has hurt, instead of helping an unrelated third party. Some of these studies found a significant influence of self-agency on the tendency to repair (Carlsmith \& Gross, 1969; de Hooge, 
Nelissen, Breugelmans, \& Zeelenberg, 2011; Freedman et al., 1967; Parkinson \& Illingworth, 2009), but others did not (Konecni, 1972; Struthers, Eaton, Shirvani, Georghiou, \& Edell, 2008). The majority of the cited studies manipulated self-agency in a social context (i.e., participants caused a goal-incongruent situation for another person) because they focused on feelings of guilt, which are thought to reflect the appraisal that one has caused interpersonal harm (Baumeister, Stillwell, \& Heatherton, 1994; Keltner \& Buswell, 1996). Regret and guilt are believed to partly overlap. Both are assumed to be characterized by the tendency to repair (Baumeister, Stillwell, \& Heatherton, 1995; Haidt, 2003; Roseman et al., 1994), but feelings of regret can arise in a broader range of situations than feelings of guilt, including non-social ones (Zeelenberg \& Breugelmans, 2008). The mixed results concerning the relation between selfagency and the tendency to repair could be due to the complex social contexts that were used to study guilt. For instance, Konecni (1972) compared a group of participants who caused someone to drop a folder with cards (self-agency) with a group that saw someone else cause it (otheragency) and observed that the first group felt embarrassed and therefore quickly disappeared from sight. In a social context, feelings of shame and fear (e.g., for angry reactions from others) and the tendency to avoid may be stronger than the tendency to repair. If so, the relation between self-agency and the tendency to repair may be more robust outside a social context. On the other hand, it could also be that the social context is necessary for obtaining this relation. The few studies that examined the role of self-agency outside a social context unfortunately measured behaviors that may stem from other motivations than the tendency to repair. For instance, information seeking may stem from the tendency to reduce uncertainty rather than the tendency to repair (Shani \& Zeelenberg, 2007). 
In Study 1, we examined the influence of the appraisal of self-agency on the tendency to repair in a non-social context, using dependent measures that were designed specifically to pick up the tendency to repair. In a multiple-trial game, participants encountered goal-congruent and goal-incongruent outcomes caused by themselves (self-agency) vs. caused by a die (circumstances-agency). We expected that the manipulation of agency would not be confounded with other appraisals, such as goal congruence or (un)fairness, because a die is a fair medium. The tendency to repair was measured via actual repair behavior after each encounter with a goalincongruent outcome and via self-reports at the end of the experiment. A schematic overview of the hypotheses is shown in Figure 1. Following appraisal theories, our first hypothesis (H1) was that there would be a causal relation between the appraisal of self-agency and the tendency to repair: Goal-incongruent outcomes caused by the self elicit a stronger tendency to repair than goal-incongruent outcomes caused by circumstances.

In addition to the causal relation between the appraisal of self-agency and the tendency to repair, Study 1 also aimed to investigate (a) the relation between the appraisal of self-agency and the feeling of regret and (b) the relation between the tendency to repair and the feeling of regret. Previous studies using scenario (Zeelenberg, van Dijk, \& Manstead, 1998) and recall methods (Zeelenberg, van Dijk, Manstead et al., 1998) have provided support for these relations. However, both methods have met with serious criticisms (Parrott \& Hertel, 1999). Therefore, we tried to replicate these findings in a carefully controlled experiment in which the appraisal of agency was manipulated with actual events and participants' feelings of regret, disappointment, and anger were collected. In line with previous studies (van Dijk \& Zeelenberg, 2002; Zeelenberg, van Dijk, \& Manstead, 1998), our second hypothesis was that goal-incongruent events caused by oneself would elicit more regret than goal-incongruent events caused by 
circumstances $(\mathrm{H} 2 \mathrm{a})$ but not more disappointment and anger $(\mathrm{H} 2 \mathrm{~b})$. Moreover, also in line with previous studies (Zeelenberg, van Dijk, Manstead et al., 1998) our third hypothesis was that the tendency to repair would be associated with feelings of regret (H3a) but not with other negative feelings such as disappointment and anger (H3b). Study 1 produced several findings that diverged from the literature. These were further examined in Study 2.

\section{Study 1}

Participants played a choice game on the computer in which they tried to win points. High scores were rewarded with lottery tickets. The game consisted of a series of trials on which the participant steered an avatar in the upper or lower road of a fork (see Figure 2). There was a piece of food at the end of each road. On self-agency trials the participant could choose a road; on circumstance-agency trials the experimenter rolled a die that determined which road the participant had to take. The participant then moved the avatar in the upper or lower road toward the piece of food. When the avatar reached the food, both pieces of food turned into points: one piece turned into ten points, the other into zero points. Goal-congruent trials were those on which the avatar had taken the road with ten points. Goal-incongruent trials were those on which the avatar had taken the road with zero points. On each goal-incongruent trial a fix button appeared. The participant could try to repair the goal-incongruent outcome by moving the avatar toward this button and pressing it a number of times. On half of the goal-incongruent trials, pressing the fix button resulted in regaining the ten points; on the other half, it did not. The participant had to decide on each goal-incongruent trial whether to repair (by moving toward the fix button) and how long to keep trying to repair (how many times to press the fix button). The number of repair responses was used as a measure for the tendency to repair. At the end of the experiment we also assessed the self-reported tendency to repair on self-agency trials and on circumstances-agency 
trials. The repair behavior in our study was not costly nor limited to a number of predetermined responses. This was done to encourage participants to respond according to their intuition rather than according to some strategy that might be based on other factors than agency (e.g., available resources).

\section{Method}

\section{Participants.}

A total of 26 students $\left(M_{\text {age }}=21 ; 12\right.$ women $)$ at Ghent University participated in the study, nineteen in return for course credits and seven in return for payment $(8 €)^{1}$.

\section{Apparatus.}

The experiment was programmed in $\mathrm{C}++$ and run in Visual Studio 6.0. It was displayed on a 19" CRT screen. The game map consisted of a two-dimensional maze with turquoise walls and grey paths, forming six horizontally positioned forks and a connection between them, that was blocked by a brown wall. The six forks were arranged in three layers of two forks (see Figure 2). If the avatar had reached the end of the sixth fork, it was automatically transported back to the first fork. Participants used the arrow keys on the keyboard to move the avatar through the maze. Participants won a lottery ticket (a scratch-and-win card) during the game. Each card yielded $25 \%$ chance of winning a money prize between one and ten thousand euro.

\section{Procedure.}

All participants were tested individually in a session of one hour. The experimenter was seated next to the participant except when the participant filled in the questionnaire. Before the start of the experiment, each participant signed an informed consent form. The experiment was a choice game consisting of 160 trials (i.e., 160 times crossing a fork). A trial started when the avatar reached the brown wall that blocked the next fork. The brown wall then disappeared and 
two food items appeared, one in the upper and one in the lower road of the fork. The participant then moved the avatar toward the start of the fork, at which time the word "ik", which is Dutch for "me" (on self-agency trials, 80 trials) or a picture of a die (on circumstances-agency trials, 80 trials) appeared in front of the avatar (see Figure 3). On self-agency trials, the participant chose a road by saying "boven" (which is Dutch for "up") or “onder" (which is Dutch for “down”). He/she then pressed ENTER to remove the word "ik" and steered the avatar in the road of his/her choice. On circumstances-agency trials, the experimenter rolled a real die with the labels B and $\mathrm{O}$ and named the outcome of the die (if B then "boven"; if $\mathrm{O}$ then "onder"). The participant pressed ENTER to remove the picture of the die and then steered the avatar in the road indicated by the die. As soon as the avatar entered one of the roads, the roads were closed off by a brown wall to prevent the avatar from going back. Our cover story provided participants with explicit instructions on how to choose a road/food during the game:

The computer determines via a very complex algorithm ${ }^{2}$ which number of points is behind which food. This algorithm is too difficult to discover, but research has shown that if people follow their intuition they perform better than according to chance level. We would like to test this hypothesis. This is why we ask you to make choices according to your intuition. Try to ask yourself each time the following question: "Which choice feels best?"

When the avatar reached the food in the upper or lower road, both food items were replaced with a number. On goal-congruent trials (60\% of the trials), the food next to the avatar was replaced with ten and the other with zero (see Figure 3), a positive sound was played, and the participant's score was increased by ten points. After the points were shown, the game froze for $500 \mathrm{~ms}$ and a new trial began. On goal-incongruent trials (40\% of the trials), the food item 
next to the avatar was replaced with zero and the other item with ten, a negative sound was played, and the score remained unchanged. The game then froze for $500 \mathrm{~ms}$ before a sideway with a fix button appeared (see Figure 3). The participant could either move the avatar toward the fix button and try to repair the goal-incongruent outcome or move the avatar toward the brown wall that blocked the next fork to start a new trial. The computer determined at random which trials were goal congruent and which trials were goal incongruent.

On half of the goal-incongruent trials, the ten points could be regained after pressing the fix button ten to thirty times. The exact number of required button presses was determined at random on each trial and was unknown to the participants (participants also did not know it would be a number between ten and thirty). If the participant pressed the fix button a number of times equal to the random number on that trial, the ten and zero points switched places, the fix button disappeared, a positive sound was played, and the participant's score was increased by ten points. After that, a new trial began. On the other half of the negative trials, the ten points could not be regained. The participant had to decide how long to keep pressing the fix button before starting the next trial (by moving the avatar toward the brown wall that blocked the next fork). The number of button presses on these trials was used as an index for the tendency to repair.

All instructions were given orally and were followed by two demonstration trials and five practice trials. The demonstration trials consisted of one goal-congruent self-agency trial and one goal-incongruent circumstances-agency trial. On the goal-incongruent trial, the experimenter demonstrated how to repair the goal-incongruent outcome (the ten points were regained after 15 button presses). The practice trials consisted of one goal-congruent and two goal-incongruent circumstances-agency trials (one repairable after 15 button presses) and two goal-incongruent self-agency trials (one repairable after 15 button presses). We informed participants that on half 
of the trials repairing would not work, irrespective of how many times they pressed the fix button. Again, we asked participants to use their intuition when deciding about the number of times to press the button. There was no time limitation for the repair responses nor a speeded response instruction. Participants were informed that the game would end after their avatar had completed 160 forks.

Participants were told they would win a lottery ticket after finishing a maze combined with a score equal to or higher than 450 points, 900 points, and 1350 points. In general, participants had to complete about ten mazes to reach a score of 450 points and twenty mazes to reach a score of 900 points. In these cases, a message appeared that the 450 or 900 threshold was reached and a lottery ticket was won. After the last trial, the total number of points was displayed (which was always below the threshold of 1350 points), together with a message that the third lottery ticket was not won.

At the end of the experiment, a questionnaire was administered in which we measured appraisals, action tendencies, and feelings separately for self-agency and circumstances-agency trials. For each trial type, we presented four scenes and asked participants to rate items on sevenpoint scales specified below. Scene 1 depicted the avatar at the start of a fork, when the word "ik" or a die appeared, and participants rated appraisals of expectancy and self-agency. For expectancy, they estimated the chance of winning ten points immediately (when the food turned into points) and eventually (at the end of the trial) on a scale ranging from 1 (very low) to 7 (very high). For self-agency, they rated the extent to which they felt they could influence the number of points they would win immediately and eventually on a scale ranging from 1 (not at all) to 7 (yes completely). 
Scene 2 depicted a zero point outcome and participants rated appraisals of goal congruence or valence $(1=$ very negative, $7=$ very positive $)$, fairness $(1=$ very unfair, $7=$ very fair), future expectancy (the likelihood of winning back the ten points after moving into the sideway, $1=$ very low, $7=$ very high), and coping potential (the extent to which the participant estimated he/she could influence the winning back of ten points; $1=$ not at all, $7=$ yes completely). We also measured feelings of regret, disappointment, and anger, as well as the tendency to repair (on scales ranging from $1=$ not at all to $7=$ yes completely). For the tendency to repair, participants indicated (a) the extent to which they were inclined to take the sideway and try to regain the ten points and (b) the extent to which they were inclined to keep trying if repairing did not immediately lead to success.

\section{Results}

The behavioral and the self-report data were analyzed via paired samples t-tests, Pearson correlation coefficients, and Steiger Z-tests. Before discussing the results for $\mathrm{H} 1$ to H3, we tested whether the agency manipulation was successful and whether it affected only agency or also other appraisal variables.

\section{Manipulation check.}

As can be seen in Table 1, self-agency trials scored significantly higher than circumstances-agency trials on the appraisal of self-agency. As expected, participants indicated they had more influence on the number of points they won on self-agency than on circumstances-agency trials. There were no differences between the self- and circumstancesagency trials for the appraisals of valence, fairness, future expectancy, coping potential and expectancy of winning ten points immediately (i.e., when the food turned into points), but there was a difference for the expectancy of winning ten points eventually (i.e., at the end of the trial). 
More specifically, participants had higher expectancies to win the ten points eventually on selfthan on circumstances-agency trials.

\section{The influence of the appraisal of agency on the tendency to repair.}

To investigate whether self-agency trials elicited a stronger tendency to repair than circumstances-agency trials (H1), we inspected repair behavior (on-line) and the self-reported tendency to repair (at the end of the experiment). We first removed all trials on which repairing was possible ${ }^{3}$ (leaving 16 self-agency and 16 circumstances-agency trials in the analysis) and all trials on which the number of repair responses deviated more than $2.5 S D$ s from the means on self-agency and circumstances-agency trials $(1.56 \% \text { of the trials })^{4}$. Subsequently, we calculated the average number of repair responses on self- and circumstances-agency trials. Dependentsamples t-tests showed that participants did not repair more on self- than on circumstancesagency trials (see Table 1). Additional analyses were conducted to investigate whether the absence of an effect could be due to a strategy of participants to press the repair button a fixed number of times on most (or all) of the trials. The within-subject range of the number of repair responses $(M=43.50 ; S D=26.43)$ as well as the within-subject standard deviation of the number of repair responses $(M=10.93, S D=6.93)$ suggested there was trial-to-trial variation in the number of repair responses participants emitted. This within-subjects standard deviation was significantly higher on the trials of interest (in which repairing was not possible) than on the remaining trials (in which repairing was possible and the repair button disappeared after 10 to 30 button presses), $M=6.26, S D=1.93), t(25)=4.07, p<.001$. Thus, the variance in repair behavior even exceeded the variance in the number of responses required to repair goalincongruent outcomes on the repair-possible trials. 
Confirming the data obtained with the behavioral measure, agency did not significantly influence the self-reported tendency to repair. Neither of the items that measured the tendency to repair revealed significant differences for self-agency and circumstances-agency trials (not confirming H1; see Table 1).

\section{The relation between the appraisal of self-agency and feelings of regret,}

\section{disappointment, and anger.}

As predicted, self-agency trials gave rise to stronger feelings of regret than circumstances-agency trials (confirming $\mathrm{H} 2 \mathrm{a}$ ) and anger did not differ significantly between selfagency trials and circumstances-agency trials (partly confirming $\mathrm{H} 2 \mathrm{~b}$ ). Contrary to the predictions, however, self-agency trials also elicited stronger feelings of disappointment than circumstances-agency trials (disconfirming $\mathrm{H} 2 \mathrm{~b}$, see Table 1).

\section{The relation between the tendency to repair and feelings of regret, disappointment,}

\section{and anger.}

We tested the hypotheses that the tendency to repair is correlated with feelings of regret (H3a) but not with feelings of disappointment or anger (H3b). Table 2 presents correlations that were calculated separately for self-agency trials, circumstances-agency trials, the difference scores between self-agency and circumstances-agency trials, and the averages across self-agency and circumstances-agency trials. A correlation between the difference scores means that the influence of agency on feelings is correlated with the influence of agency on the tendency to repair. Although there was no main effect of agency on the tendency to repair in previous analyses, it could be that there is interindividual variation in the effect of agency on the tendency to repair that is related to variation in the effect of agency on feelings. Again, we used both a behavioral measure and a self-report measure for the tendency to repair. Before calculating 
correlations between the self-reported tendency to repair and feelings, we summated the two items used to measure the tendency to repair into one scale.

The correlations are presented in Table 2. Contrary to the predictions, none of the correlations between regret and the tendency to repair were significant (neither for the selfreports nor for the behavioral measures; not confirming $\mathrm{H} 3 \mathrm{a}$ ). On the other hand, all correlations between disappointment and the tendency to repair were significant (or marginally so) and a number of correlations between anger and the tendency to repair were significant (disconfirming $\mathrm{H} 3 \mathrm{~b})$. Steiger Z-scores were calculated to investigate whether the correlations between the tendency to repair and disappointment and the tendency to repair and anger were significantly higher than the correlations between the tendency to repair and regret. As can be seen in Table 2, a subset of the correlations for disappointment differed significantly from the correlations for regret. The correlations between disappointment and the tendency to repair on self-agency trials were higher than the correlations between regret and the tendency to repair on these trials: behavioral measure $^{5}, z=2.12, p=.034$, self-report measure, $z=1.170, p=.090$. Similarly, the correlations between the difference in disappointment on self- and circumstances-agency trials and the difference in the tendency to repair on self- and circumstances-agency trials was higher than the same correlations for regret: behavioral measure, $z=1.93, p=.053$, self-report measure, $z=2.20, p=.028$. Finally, the correlations for disappointment across trials and the tendency to repair across trials were marginally higher for disappointment than for regret for the behavioral measure, $z=1.94, p=.053$ (but not for the self-report measure, $z=1.14, p=.25$ ). The correlations between disappointment and the tendency to repair on circumstances-agency trials were not significantly different from the correlations between regret and the tendency to repair on these trials, $z \mathrm{~s}<1.31, p>.19$ (see Table 2). Contrary to the pattern for disappointment, none 
of the correlations for anger were significantly different from those for regret, all $z \mathrm{~s}<1.51, p \mathrm{~s}>$ .13 .

\section{Discussion}

Study 1 did not provide support for a causal influence of the appraisal of self-agency on the tendency to repair (not confirming H1). Both the self-report and the behavioral measures suggested that participants were equally motivated to repair goal-incongruent outcomes caused by themselves as those caused by circumstances. One could argue that this finding is not a genuine null finding, but rests on methodological shortcomings of Study 1. For instance, the reward value of the repair responses may have been too high, causing the tendency to repair to be at ceiling, leaving little room for agency to influence the tendency to repair. This explanation is unlikely, however, given the within-subject variation in the amount of repair responses and the significant correlations between the difference in repair behavior on self- vs. circumstancesagency trials and the difference in feelings of disappointment on self- vs. circumstances-agency trials. These correlations suggest that our operationalization of agency was capable of producing meaningful within-subject differences in the tendency to repair. Our results thus provide a first indication that other variables (such as the intensity of disappointment) may be a better predictor of the tendency to repair than the appraisals of self- and/or circumstances-agency.

In addition, our study contradicted several findings of previous research. First, we found that goal-incongruent events elicited both stronger feelings of regret and stronger feelings of disappointment when they were caused by oneself than when they were caused by circumstances (confirming H2a, disconfirming H2b). Zeelenberg, van Dijk, and Manstead (1998) manipulated agency via scenarios and showed that goal-incongruent events caused by oneself elicited more regret but less disappointment than goal-incongruent events caused by circumstances. Second, 
we found that the tendency to repair was related to the intensity of disappointment but not to the intensity of regret (disconfirming H3a and H3b). A study by Zeelenberg, van Dijk, Manstead, and van der Pligt (1998) showed the exact opposite pattern. In sum, Study 1 provided support for one of the five hypotheses (H2a) only.

\section{Study 2}

A first aim of Study 2 was to further investigate the relation between self-agency and the tendency to repair. Study 1 did not provide support for the idea that the appraisal of self-agency increases the tendency to repair. However, self-agency and the tendency to repair may be related in other ways. We explored the possibility that the appraisal of self-agency and the tendency to repair may be related in an indirect way, via the appraisal of coping potential. This idea builds on a functional view of emotions and action tendencies (Camille et al., 2004; Lazarus, 1991; Roseman et al., 1996; Wortman \& Brehm, 1975) which proposes that action tendencies are responses to the environment that can be understood from their benefits to the organism. According to this view, the tendency to repair will be activated when it is possible and functional to repair, thus when coping potential is high. In daily life, self-agency and high coping potential often may co-occur: Goal-incongruent events caused by oneself typically may be easier to cope with (i.e., undo or repair) than goal-incongruent events caused by others or circumstances. For example, when you end a relationship with someone (self-agency), you have a higher chance of fixing it again (coping potential is high) than when the other person ends it (other-agency, coping potential is low). High coping potential has been shown to increase active problem-solving behaviors that could be seen as expressions of the tendency to repair (Galinsky, Gruenfeld, \& Magee, 2003; Mikulincer, 1988, 1994; Wortman \& Brehm, 1975). In Study 1, the natural cooccurrence between the appraisal of self-agency, the appraisal of coping potential, and the 
tendency to repair may have been artificially pulled apart by carefully matching the self-agency and circumstances-agency trials for coping potential (see Table 1). In Study 2, we investigated the possibility that self-agency and the tendency to repair co-occur because both are related to coping potential, using the method of autobiographical recall.

A second aim of Study 2 was to investigate three possible explanations for the inconsistencies between our results and those obtained in previous studies concerning the relation between regret and the tendency to repair. In these previous studies, participants recalled an event in which they felt regret or disappointment and indicated the extent to which they had the tendency to repair. The results of these studies showed that recalled regret was associated with a stronger tendency to repair than other negative feelings, such as disappointment (Roseman et al., 1994; Zeelenberg, van Dijk, Manstead, et al., 1998). A first difference is that in previous studies the tendency to repair was often measured using items that implied self-agency. For instance, Roseman et al. (1994) used items such as "to what extent did you feel like correcting a mistake" (implying that the person made a mistake), "want to get a second chance" (implying that the person failed the first time), and "want to improve your performance" (implying that the outcome was caused by the person's previous performance). Given the close relation between regret and self-agency, the relation between regret and the tendency to repair may have been overestimated by measuring the tendency to repair via items that implied self-agency. Zeelenberg, van Dijk, Manstead, and van der Pligt (1998) used several items of Roseman et al. (1994) that implied self-agency, with the exception of one item: "to what extent did you want to undo the event?" Recalled regret was associated with a stronger tendency to undo the event than recalled disappointment $\left(\mathrm{g}_{\mathrm{s}}=0.40\right.$, a small to medium effect). This finding does suggest that a person feeling regret is more motivated to repair a goal-incongruent outcome than a person 
feeling disappointment. In Study 2, we tried to replicate and extend the findings of Zeelenberg, van Dijk, Manstead, and van der Pligt (1998). We measured the tendency to repair with two sets of items: one set that referred to the appraisal of self-agency (e.g., "to what extent did you want a second chance") and another set that did not refer to the appraisal of self-agency (e.g. "to what extent did you have the tendency to undo the event"). Similar to Zeelenberg, van Dijk, Manstead, and van der Pligt (1998), we compared the tendency to repair for participants who recalled an instance of regret and ones who recalled an instance of disappointment. We hypothesized that there would be a difference between regret and disappointment for items referring to self-agency and a significantly smaller difference for items not referring to self-agency (H4). A power analysis suggested that to obtain a significant effect for the items not referring to self-agency ( $\alpha$ $=.05, \beta=0.80$ ), we needed a sample of at least 200 participants (Zeelenberg, van Dijk, Manstead et al., 1998).

A second difference is that previous studies compared instances of regret and disappointment across participants, whereas we used an individual differences approach (correlating feelings of regret/disappointment with the tendency to repair). In Study 2, we used both approaches: As described above, we used the same approach as Zeelenberg, van Dijk, Manstead, and van der Pligt (1998). In addition, we used the individual differences approach of our own Study 1 and investigated whether the intensity of regret or the intensity of disappointment was a better predictor of the tendency to repair. To address this question, we asked all participants (both those who recalled an instance of regret and those who recalled an instance of disappointment) to indicate the intensity of regret and disappointment. Study 1 suggested that the intensity of disappointment would be a significant predictor of the tendency to repair (H5) but not the intensity of regret (H6). 
A third difference between our Study 1 and previous studies is that the latter investigated naturally occurring regret and disappointment, whereas we used a carefully controlled experimental setting. In real life, the circumstances giving rise to regret may be correlated with the circumstances giving rise to the tendency to repair, but these circumstances may not have been present in Study 1. For instance, as argued before, regret may follow from an appraisal of self-agency and the tendency to repair may follow from an appraisal of coping potential and both appraisals may often co-occur. The current Study 2 investigated naturally occurring regret and disappointment to keep this feature similar to other studies. To investigate the hypothesized sets of co-occurrences, we estimated the strengths of all possible direct and indirect relations between appraisals (self-agency and coping potential), action tendencies (the tendency to repair), and feelings (regret and disappointment), using structural equation modeling (SEM). The investigated model is presented in Figure 4. We are aware that SEM does not allow us to test causal relations among the variables. Therefore all our hypotheses were framed in terms of "variable $\mathrm{X}$ is related to/ (statistically) predicts variable $\mathrm{Y}$ " and not in terms of causality. Because we were interested in predicting the values of some variables (i.e., the tendency to repair) and not others (i.e., the appraisal of self-agency and coping potential), the former were entered as outcomes and the latter as predictors. Feelings of regret and disappointment were sometimes entered as predictors and sometimes as outcomes. We tested the idea that self-agency is not directly related to the tendency to repair $(\mathrm{H} 7)$, but that self-agency is related to coping potential (H8), and that coping potential is related to the tendency to repair (H9). In addition, we tested whether the appraisal of self-agency is positively related to feelings of regret $(\mathrm{H} 10)$.

Finally, we examined the remaining relations between appraisals and feelings. We investigated if we could replicate the relation between self-agency and disappointment obtained 
in Study 1 (H11) and explored the relations between coping potential and regret (H12) and between coping potential and disappointment (H13).

\section{Method}

\section{Participants.}

A total of 659 psychology students at Ghent University were contacted via email to fill in an online questionnaire study on regret or disappointment in return for participation in a prize lottery. After a first call for responses and a reminder after two and five weeks, 114 responses were obtained for the regret questionnaire (response rate $34.55 \%$ ) and 116 for the disappointment questionnaire (response rate $35.26 \%$ ). Four non-native Dutch speaking participants were excluded because their level of Dutch (as apparent from their written answers to four open questions) was judged as insufficient by two independent raters. In total 114 regret respondents (21 men) and 112 disappointment respondents (22 men) remained in the study $\left(M_{\text {age }}=18.95, S D\right.$ =3.67). All answers were collected and stored anonymously.

\section{Procedure.}

Participants were asked to fill in a 15 minute online questionnaire (administered via Limesurvey) about regret or disappointment as part of a large-scale research project on emotions. Participants were randomly assigned to the regret or disappointment questionnaire. On the first page of the questionnaire, participants were informed about the general aim of the study and about data confidentiality. The questionnaire contained more items than needed for the present study. We briefly mention the content of the irrelevant items to sketch the measurement context of the items under study.

The questionnaire started with a number of demographic questions (sex, age, nationality, and native language). Subsequently, participants were asked to take a few minutes to recall an 
event in their life in which they felt regret (in the regret condition) or disappointment (in the disappointment condition). The instructions encouraged participants to recall an event that was specific in time and space. Participants wrote down a short description of the recalled event and indicated the approximate date of the event on a calendar. They also rated the intensity of regret (in the regret condition) or disappointment (in the disappointment condition) at the time of the event as well as at the time of recall on two scales ranging from 1 (completely not intense) to 9 (very intense).

Subsequently, several appraisals, ruminative thoughts (e.g., counterfactual thoughts), action tendencies, and behaviors were measured. Items were rated on scales ranging from 1 (not at all) to 9 (yes completely), unless stated otherwise. In respective order, the following appraisals were measured: novelty, expectancy, goal congruence for yourself and for others, valence for yourself and for others, prevention focus, promotion focus, agency, coping potential, future expectancy, intrinsic controllability, norm violation, and uncertainty. The appraisal of selfagency was measured via two items $(\alpha=.65)$. One item asked participants to briefly describe the cause of the event and to indicate the extent to which the event was a consequence of their own behavior or choices ${ }^{6}$. The other item asked participants to rate the extent to which they had control over the occurrence of the event. The appraisal of coping potential was measured via four items $(\alpha=.77)$ : Participants rated whether (a) they were able to change the event after its occurrence, (b) they were able to undo the event, (c) they were able to improve the situation, and (d) their behavior determined whether the event could still change.

Action tendencies were measured by asking participants about particular thoughts about actions, wishes about action outcomes, and action tendencies that accompanied the feeling of regret and disappointment. Items of the latter type were preceded by a short introductory 
paragraph: "The feeling of regret (disappointment) is sometimes accompanied by the tendency to perform a particular behavior. Indicate to which extent you had the tendency to behave in a particular way" (the word "tendency" was underlined). In total, three types of action tendencies were measured: The tendency to repair (6 items), the tendency to avoid (11 items), and the tendency to be passive (4 items). The tendencies to avoid and to be passive were not analyzed in the current study. The tendency to repair was measured with two sets of items, some of which were adopted from previous studies (Roseman et al., 1994; Zeelenberg, van Dijk, Manstead et al., 1998). Items in the first set did not involve a confound of the tendency to repair with selfagency ( $\alpha=.74$ ), asking to which extent participants had the tendency to (a) change the situation, (b) improve or rectify the situation, and (c) undo the event. Items in the second set of items did involve a confound of the tendency to repair with self-agency $(\alpha=.62)$, asking to which extent participants (a) thought about how they would handle the situation differently next time, (b) wished they could turn back time, and (c) wanted a second chance. The latter items are confounded with self-agency because only if the participant's behavior influenced the occurrence of the situation, it makes sense to think about handling the situation differently next time, turning back time, and wanting a second chance. For instance, if one believes that the event was caused by circumstances or others, turning back time is pointless because the same event is very likely to occur again.

After completing the items on action tendencies, participants rated their actual behavior in the situation: repairing ( 3 items), avoidance ( 4 items), passivity ( 3 items), apologizing ( 1 item), and aggression (1 item). In the current study, we focused on action tendencies rather than actual behavior to avoid the criticism that it is obvious that people only perform repair behavior when it is physically possible (i.e., in situations with high coping potential). Finally, participants 
rated the intensity of feelings of fear, sadness, anger, guilt, happiness, powerlessness, energy, restlessness, calmness, despair, frustration, shame, relief, and regret or disappointment ${ }^{7}$. In the disappointment condition, participants were additionally asked to indicate whether the episode that they had recalled could be categorized as disappointment over an outcome or in a person ${ }^{8}$.

The model was fitted on the entire sample (collapsing recalled regret and disappointment) via structural equation modeling using the Lavaan 0.4-12 package in R (Rosseel, 2012). Three variables were treated as latent variables: the appraisal of self-agency ( 2 items), the appraisal of coping potential (4 items), and the tendency to repair (3 items, not confounded with self-agency). The intensities of regret and disappointment were each measured with a single item. The goodness-of-fit was evaluated via four fit indexes: Chi-square (Chi-square divided by the degrees of freedom should be $<2$ ), the comparative fit index (CFI, should be $>.95)$, the root-meansquare error of approximation (RMSEA, should be $<.06$ ), and the standardized root-meansquare residual (SRMR, should be <.09; Hu \& Bentler, 1999). Standardized parameter estimates are reported.

\section{Results}

We first discuss the results for $\mathrm{H} 4$ and then turn to the model fitting to test H5 to H13.

\section{Hypothesis 4.}

Before testing Hypothesis 4, we ran an exploratory factor analysis (with varimax rotation) on the six repair items to investigate whether the confounded and non-confounded items loaded onto different factors. The factor analysis confirmed the hypothesized two-factor structure: A first factor with high loadings for the non-confounded items (.70 to .87) and low loadings for the confounded items (.06 to .26) and a second factor with high loadings for the 
confounded items (.69 to .81) and low loadings for the non-confounded items (.05 to .23). This suggests that the confounded and non-confounded items reflected different constructs.

Next, we tested whether the difference between recalled regret and disappointment with respect to the tendency to repair was stronger for items that confounded self-agency and the tendency to repair than for items that did not (H4). First, the confounded and non-confounded items were aggregated into two variables. Second, a mixed models ANOVA was run to test for an interaction between item type (confounded vs. non-confounded) and condition (recalled regret vs. recalled disappointment). This interaction was highly significant, $F(1,224)=15.63, p<.001$, $\eta_{\mathrm{p}}^{2}=.065$. As expected, participants in the regret condition reported a stronger tendency to repair $(M=7.35, S D=1.72)$ than participants in the disappointment condition $(M=6.26, S D=2.04)$, $t(224)=4.34, p<.001$, on items that were confounded with self-agency. However, on items that were not confounded with self-agency, participants in the regret condition reported an equally strong tendency to repair $(M=5.94, S D=2.15)$ than participants in the disappointment condition $(M=6.03, S D=2.20), t(224)=-0.31, p=.76$. Independent samples t-tests showed that all confounded items presented the expected difference. Recalled regret was accompanied by more thoughts on handling the situation differently next time (difference $=0.89, C I_{.95}=0.20,1.58$; $t(224)=2.54, p=.012)$, with wanting to turn back time (difference $=1.68, C I_{.95}=1.04,2.32 ;$ $t(224)=5.15, p<.001)$, and with wanting a second chance $\left(\right.$ difference $=0.70, C I_{.95}=0.04,1.36$; $t(224)=2.08, p=.039)$ than recalled disappointment (see Table 3). There were no differences for any of the non-confounded items, i.e., the tendency to change the situation (difference = $\left.0.47, C I_{.95}=-1.15,0.22 ; t(224)=1.35, p=.18\right)$, the tendency to improve or rectify the situation (difference $\left.=-0.06, C I_{.95}=-0.76,0.64 ; t(224)<1\right)$, and the tendency to undo the event 
(difference $=0.26, C I_{.95}=-0.46,0.98 ; t(224)<1$, see Table 3 ). For the SEM we used the nonconfounded items only.

\section{Hypotheses 5 to 13.}

Before fitting the model, multivariate normality for the data was inspected by plotting the quantiles of the Mahalanobis transformed data against the quantiles of a chi-square distribution with 11 degrees of freedom. The data points showed a systematic deviation from the expected distribution. We therefore applied the Satorra-Bentler correction (Hu, Bentler, \& Kano, 1992; Satorra \& Bentler, 1994; scaling factor 1.028).

We first investigated whether the model (Figure 4) reproduced the data sufficiently well (i.e., whether the absolute fit of the model was acceptable). Fit indexes indicated a good model fit, $\chi^{2}(37)=61.50, p=.007 ; \mathrm{CFI}=.956, \mathrm{RMSEA}=.054 ;$ SRMR $=.047$. Subsequently, we inspected the estimates for each of the discussed relations.

First, we examined the hypotheses pertaining to the relations between the feelings (disappointment and regret) and the tendency to repair (H5 and H6). Replicating the results of Study 1, the intensity of disappointment was a significant predictor of the tendency to repair (confirming H5), $z=3.37, p=.001$, and the intensity of regret was not (confirming H6), $z=1.38$, $p=.17$. To investigate whether disappointment predicted the tendency to repair significantly better than regret, we investigated the fit of a constrained model that had the same structure as the model in Figure 4, but with the additional constraint that the parameter of the relation between regret and the tendency to repair had to be equal to the parameter of the relation between disappointment and the tendency to repair. If the fit of the constrained model is statistically lower than that of the unconstrained model (in which these parameters are estimated separately and thus are allowed to differ), one can conclude that the relations are statistically 
different. There was a trend toward a better fit for the unconstrained model, $\chi_{D}(1)=3.29, p=$ .0696 (scaled difference test; Satorra, 2000; Satorra \& Bentler, 2010), suggesting that disappointment was a better predictor of the tendency to repair than regret.

Second, we examined the hypotheses pertaining to the relations between the appraisals (self-agency and coping potential) and the tendency to repair (H7 and H9) and the relation among these appraisals (H8). As predicted, we found no direct relation between the appraisal of self-agency and the tendency to repair (confirming H7), $z=-0.36, p=.72$, but we did find a direct positive relation between coping potential and the tendency to repair (confirming H9), $z=$ $2.78, p=.005$. The relation between the appraisals of self-agency and coping potential was positive and significant (confirming H8), $z=4.77, p<.001$. The strength of the indirect relation between self-agency and the tendency to repair was .16 (equaling the product of the two direct relations, i.e., .49 and .33). When fitting the same model without the arrow between coping potential and the tendency to repair, a significant relation between self-agency and the tendency to repair did emerge ( $\beta=0.19, z=2.00, p=.046)$. This confirms that the relation between selfagency and the tendency to repair can be explained by a relation between self-agency and coping potential and a relation between coping potential and the tendency to repair.

Third, we examined the hypotheses pertaining to the relation between appraisals (selfagency and coping potential) and feelings (regret and disappointment; H10 to H13). The appraisal of self-agency was directly related to regret (confirming H10 and replicating the result of Study 1), $z=3.39, p=.001$, but unrelated to disappointment (disconfirming H11 and not replicating the result of Study 1), $z=-1.07, p=.28$. The appraisal of coping potential was not related to regret $(\mathrm{H} 12), z=-.619, p=.54$, nor to disappointment $(\mathrm{H} 13), z=1.35 p=.18$. 
Finally, we investigated whether the relation between regret and the tendency to repair was significant when fitting the same model without the direct relations between the appraisals (self-agency and coping potential) and the tendency to repair. In this model disappointment again was a strong predictor of the tendency to repair $(\beta=.27, z=3.63, p<.001)$, and the relation between regret and the tendency to repair was marginally significant $(\beta=.14, z=1.89, p=.059)$. This suggests that the relation between the feeling of regret and the tendency to repair can be explained by the relation between regret and the appraisal of self-agency, the relation between self-agency and coping potential, and the relation between coping potential and the tendency to repair.

\section{Discussion}

Study 2 further examined the relations between the appraisal of self-agency, the tendency to repair, and regret. It supported the idea that the appraisal of self-agency and the tendency to repair co-occur because both are related to the appraisal of coping potential ( $\mathrm{H} 7$ to $\mathrm{H} 9)$. In addition, Study 2 provided novel insights on the relation between regret and the tendency to repair. In Study 1, we did not replicate the relation between regret and the tendency to repair found in previous studies (Roseman et al., 1994; Zeelenberg, van Dijk, Manstead, et al., 1998). We investigated whether the results of our study diverged from the literature because of (a) different ways of measuring the tendency to repair (confounded vs. not confounded with selfagency), (b) different designs (individual differences approach vs. comparison across individuals), and (c) different settings (laboratory vs. real life). First, contrary to the findings of Zeelenberg, van Dijk, Manstead, et al. (1998), we found that regret and disappointment differed only with regard to the tendency to repair on items that confounded the tendency to repair with self-agency. In their study, the difference between regret and disappointment on non-confounded 
items yielded an effect size of 0.40 , whereas we observed a (maximum) effect size of 0.10 . On the other hand, the difference between regret and disappointment for confounded items yielded an effect size of 0.26 to 0.60 . The predominant use of confounded items may thus have contributed to the overestimation of the relation between regret and the tendency to repair.

Second, using an individual differences approach similar to Study 1, we replicated the result that disappointment was more strongly related to the tendency to repair than regret (confirming H5 and H6). Thus, although comparing regret and disappointment across individuals does not yield significant differences with regard to the tendency to repair (on the nonconfounded items), individual differences in the tendency to repair were more strongly related to individual difference in disappointment than to individual differences in regret.

Third, we found support for the idea that the conditions for regret (i.e., the appraisal of self-agency) naturally co-occur with the conditions for the tendency to repair (i.e., the appraisal of coping potential). This may explain why studies sampling from real-life experiences show a different pattern than lab studies in which appraisal variables are manipulated in isolation. The spurious relation between regret and the tendency to repair via self-agency and coping potential explains how regret may naturally co-occur with the tendency to repair without being a good predictor of the tendency to repair.

\section{General Discussion}

Our aim was to investigate the relation between the appraisal of self-agency and the tendency to repair. In addition, we investigated how each was related to regret and to other negative feelings. In Study 1, we experimentally manipulated agency (self vs. circumstances) and measured the tendency to repair as well as feelings of regret, disappointment, and anger. In 
Study 2, participants recalled an event in which they felt regret or disappointment and rated their appraisals and action tendencies.

In the introduction we suggested that the appraisal of self-agency and the tendency to repair could be (a) causally related, (b) temporally related without a causal relation, or (c) semantically related without actually co-occurring. Neither of our studies provided support for a causal relation. In Study 1, a careful experimental manipulation of agency (self vs. circumstances) did not lead to a difference in the tendency to repair (failing to confirm H1). In Study 2 , there was no direct relation between the appraisal of self-agency and the tendency to repair. Study 2 did provide support for a temporal or semantic relation between the appraisal of self-agency and the tendency to repair, via the appraisal of coping potential: Self-caused events tended to go together with more coping potential (confirming H8) and coping potential was positively related to the tendency to repair (confirming H9). Because Study 2 relied on selfreports, we cannot determine whether these relations reflect a real-world temporal co-occurrence or a semantic relation that only exists in people's mind.

Our studies also examined the relation between the appraisal of self-agency and the feeling of regret as well as other negative feelings. Previous studies suggested that regret can be differentiated from anger, disappointment, and fear, because it reflects the appraisal of selfagency. Our studies confirmed that regret is related to the appraisal of self-agency. In Study 1, participants indicated they felt more regret in situations caused by themselves than in situations caused by circumstances (confirming $\mathrm{H} 2 \mathrm{a}$ ) and in Study 2, there was a positive relation between the appraisal of self-agency and regret (confirming H10). We also found evidence for a relation between self-agency and disappointment, but only in Study 1. One possible explanation for this pattern of findings is that self-agency relates to disappointment in certain contexts (e.g., 
achievement in general or game performance specifically), but not in others (e.g., human relations). The relation between self-agency and regret may be more context free. Another possibility is that this difference is due to other procedural differences between Study 1 and 2 . For instance, the time lag between emotional experience and measurement was very short in Study 1 (a couple of minutes at most), but long in Study 2 (the median lag between event and recall was 360 days). Other procedural differences that may have played a role are study design (experimental in Study 1 vs. correlational in Study 2) and ecological validity (low in Study 1 vs. high in Study 2).

Finally, we investigated the relation between the tendency to repair and feelings of regret and disappointment. Neither of our studies provided support for the idea that the tendency to repair more often co-occurs with feelings of regret than with other negative feelings $(\mathrm{H} 3 \mathrm{a}, \mathrm{H} 3 \mathrm{~b}$, $\mathrm{H} 4, \mathrm{H} 5$, and H6). It is important to note that the current data pattern does not imply that feelings of regret rarely co-occur with the tendency to repair, nor that the tendency to repair uniquely relates to disappointment (instead of uniquely to regret). In Study 2 (H4), participants who recalled an instance of regret and those who recalled an instance of disappointment reported an equally strong tendency to repair. In both Study 1 and 2, however, the intensity of disappointment was a better predictor of the tendency to repair than the intensity of regret. We could explain the latter finding by comparing the appraisal patterns that give rise to (a) the tendency to repair and (b) feelings labeled as regret and disappointment. With regard to "(a)", we propose that all events appraised as goal incongruent lead to the tendency to repair. In addition, we observed in our studies that this tendency is increased when coping potential is appraised as high (H9), but not when the situation is appraised as caused by oneself (H1, H7). Thus, we propose that the tendency to repair is elicited by an appraisal pattern of goal incongruence plus 
high coping potential. With regard to "(b)", it is reasonable to assume that feelings of regret and disappointment both arise in situations appraised as goal incongruent. Our studies suggest that regret also is related to the appraisal of self-agency $(\mathrm{H} 2 \mathrm{a}, \mathrm{H} 10)$ but not to the appraisal of coping potential (H12), whereas disappointment is not systematically related to either of these appraisals (H2b, H11, H13). The latter fits nicely with the idea that the word disappointment is used in a broader range of goal-incongruent situations than the word regret (Zeelenberg, van Dijk, Manstead et al., 1998). Taking "(a)" and "(b)" together, we suggest that the feeling of disappointment (related to an appraisal of goal incongruence) is a better predictor of the tendency to repair (related to an appraisal pattern of goal incongruence plus high coping potential) than the feeling of regret (related to an appraisal pattern of goal incongruence plus self-agency) because regret is related to an appraisal variable that is irrelevant to the tendency to repair (self-agency) and any variation in the feeling of regret due to this variable will not be predictive of the tendency to repair. Future studies may examine whether feelings of guilt (related to an appraisal pattern of goal incongruence for others plus self-agency; Zeelenberg \& Breugelmans, 2008), can be used to predict the tendency to repair.

Our data were not supportive of the idea that each specific feeling, such as regret and disappointment, reflects one unique action tendency, as the feeling-is-for-doing account (Zeelenberg \& Pieters, 2006) seems to suggest. The finding that both regret and disappointment relate to the same action tendency (to repair) seems more compatible with theories that argue against the existence of one-to-one relations between feelings and action tendencies, such as contemporary appraisal theories (see Moors, in press) and psychological constructivist views (e.g., Russell, 2009). Our studies did reveal recurrent patterns of relations between appraisals and feelings (e.g., self-agency and regret) and between action tendencies and feelings (e.g. the 
tendency to repair and disappointment). Thus, the present data pattern suggests that regularities do exist, but that one should be careful with assuming strict one-to-one relations between some of the components of emotions.

Our studies do not argue against the view described in the introduction that regret is related to particular counterfactual thoughts, mental time travel, and vivid simulations of alternative actions. The appraisal of self-agency may cause people to focus on their own behavior and think about how things could have been different if they had made other choices. Similarly, regret may relate to those cognitions that help a person avoid similar mistakes in the future (Baumeister, Vohs, DeWall, \& Zhang, 2007; Zeelenberg et al., 2000). The experience of regret may thus relate to qualitative behavioral changes in future choice situations (i.e., choosing those options that minimize regret). Support for this idea has been obtained in research showing that choice behavior heavily depends on anticipated regret (Zeelenberg \& Beattie, 1997; Zeelenberg, Beattie, vanderPligt, \& deVries, 1996; Zeelenberg \& Pieters, 2004b).

Our studies have a number of limitations that could be addressed in future research. First, the data of Study 1 and 2 were collected in a sample of Dutch speaking psychology students. The limitations concerning this sample are twofold. First, emotional experiences that are labeled in Dutch as regret ("spijt") and disappointment ("teleurstelling") may not be the same as those in English and other languages. A replication across other language groups therefore seems crucial. On the other hand, several of the original studies on regret and disappointment also used a Dutch sample (van Dijk \& Zeelenberg, 2002; Zeelenberg, van Dijk, Manstead et al., 1998), which makes our studies comparable to these studies at least. Second, although the program for first year psychology students at Ghent University does not contain any intensive courses on emotions, one could argue that psychology students more often think about their emotions than 
other students or non-students. Therefore, any differences between emotions that are so similar as regret and disappointment may be inflated by the fact that our participants elaborate more on their emotions than the average person (Tugade, Fredrickson, \& Barrett, 2004).

A second potential limitation is that the manipulation of agency in Study 1 may have lacked the strength to evoke differences in the tendency to repair. One could argue that the participants in Study 1 simply guessed which fruit would yield ten points and did not actually feel like "agents" on the self-agency trials. In this respect it is important to note that (a) the manipulation check showed that our manipulation of agency was successful and did produce significant differences in feelings of regret, (b) Study 2 replicated the pattern of results of Study 1, refuting the idea that this pattern of findings is limited to laboratory and/or guessing situations, and (c) the television quiz example of the introduction suggests that even when people make a guess, they still can see themselves as the cause of an outcome and experience regret when this outcome is negative. Future studies may investigate whether other experimental procedures (e.g., in which the occurrence of goal-congruent outcomes is governed by a set of rules that participants discover during the experiment) can yield stronger appraisals of self-agency and may generate different findings.

A third limitation is that the structural equation modeling of Study 2 informs us about the existence of relations between variables, but not about the (causal) nature of these relations. In Study 1, we did find support for a causal relation between the appraisal of self-agency and the feeling of regret, but several questions remain about other relations examined in Study 2. For instance, it is unclear whether the appraisals of self-agency and coping potential merely co-occur or whether they may be causally related. Study 2 also did not provide information about the (causal) nature of the relation between the appraisal of coping potential and the tendency to 
repair, but previous research has shown that the appraisal of coping potential increases behaviors that can be seen as manifestations of the tendency to repair (Galinsky et al., 2003; Mikulincer, 1988, 1994; Wortman \& Brehm, 1975).

Our studies hint at new avenues for research on the relation between the appraisal of selfagency and the tendency to repair. The studies cited in the introduction suggest that in a social context there might be a causal relation between the appraisal of self-agency and the tendency to repair. These studies show that people put more effort in repairing a goal-incongruent event for another person when they caused it themselves (Carlsmith \& Gross, 1969; de Hooge et al., 2011; Parkinson \& Illingworth, 2009, but see Konecni, 1972). The relation between self-agency and the tendency to repair may thus be moderated by the extent to which an event is negative for oneself or for others. Other potential moderators are the likability of the other person, the presence of others, and the appraisal of intentionality (i.e., whether or not the agent had the intention of causing the outcome). Future studies could also address the influence of the cost of repairing. In Study 1 repairing was not very costly. It did not consume much energy or time. One could argue that in real life, repair behavior often does require a lot of energy, and that participants would respond differently if repairing would be more costly: They might, for instance, be more reluctant to repair a negative outcome caused by circumstances.

To conclude, our studies suggest that an appraisal of self-agency increases feelings of regret but not the tendency to repair. They further suggest that feelings of disappointment are a better predictor of the tendency to repair than feelings of regret. Our studies go against the prevailing idea that a person who feels disappointment is less motivated to repair a negative outcome than a person who feels regret. 


\section{References}

Baumeister, R. F., Stillwell, A. M., \& Heatherton, T. F. (1994). Guilt: An interpersonal approach. Psychological Bulletin, 115, 243-267. doi:10.1037//0033-2909.115.2.243

Baumeister, R. F., Stillwell, A. M., \& Heatherton, T. F. (1995). Personal narratives about guilt: Role in action control and interpersonal relationships. Basic and Applied Social Psychology, 17, 173-198. doi:10.1207/s15324834basp1701\&2_10

Baumeister, R. F., Vohs, K. D., DeWall, C. N., \& Zhang, L. Q. (2007). How emotion shapes behavior: Feedback, anticipation, and reflection, rather than direct causation. Personality and Social Psychology Review, 11, 167-203. doi:Doi 10.1177/1088868307301033

Camille, N., Coricelli, G., Sallet, J., Pradat-Diehl, P., Duhamel, J. R., \& Sirigu, A. (2004). The involvement of the orbitofrontal cortex in the experience of regret. Science, 304, 11671170.

Carlsmith, J. M., \& Gross, A. E. (1969). Some effects of guilt on compliance. Journal of Personality and Social Psychology, 11, 232-239. doi:10.1037/h0027039

Cialdini, R. B., Darby, B. L., \& Vincent, J. E. (1973). Transgression and altruism: A case for hedonism. Journal of Experimental Psychology, 9, 502-516. doi:10.1016/00221031(73)90031-0

Clark, L., Crooks, B., Clarke, R., Aitken, M. R. F., \& Dunn, B. D. (2012). Physiological responses to near-miss outcomes and personal control during simulated gambling. Journal of Gambling Studies, 28, 123-137. doi:10.1007/s10899-011-9247-z

Clark, L., Lawrence, A. J., Astley-Jones, F., \& Gray, N. (2009). Gambling near-misses enhance motivation to gamble and recruit win-related brain circuitry. Neuron, 61, 481-490. doi:10.1016/j.neuron.2008.12.031

Cunningham, M. R., Steinberg, J., \& Grev, R. (1980). Wanting to and having to help: Separate motivations for positive mood and guilt-induced helping. Journal of Personality and Social Psychology, 38, 181-192. doi:10.1037//0022-3514.38.2.181

de Hooge, I. E., Nelissen, R. M. A., Breugelmans, S. M., \& Zeelenberg, M. (2011). What is moral about guilt? Acting "Prosocially" At the disadvantage of others. Journal of Personality and Social Psychology, 100, 462-473. doi:10.1037/A0021459

de Rivera, J. H. (1977). A structural theory of the emotions (Vol. 40): New York: International Universities Press.

Freedman, J. L., Wallington, S. A., \& Bless, E. (1967). Compliance without pressure: The effect of guilt. Journal of Personality and Social Psychology, 7, 117-124. doi: $10.1037 / \mathrm{h} 0025009$

Frijda, N. H. (1986). The emotions: Cambridge: Cambridge University Press.

Frijda, N. H., Kuipers, P., \& ter Schure, E. (1989). Relations among emotion, appraisal, and emotional action readiness. Journal of Personality and Social Psychology, 57, 212-228. doi:10.1037//0022-3514.57.2.212

Galinsky, A. D., Gruenfeld, D. H., \& Magee, J. C. (2003). From power to action. Journal of Personality and Social Psychology, 85, 453-466. doi:10.1037/0022-3514.85.3.453

Haidt, J. (2003). The moral emotions. In R. J. Davidson, K. R. Scherer \& H. H. Goldsmith (Eds.), Handbook of affective sciences (pp. 852-870): Oxford: Oxford University Press.

Hu, L. T., Bentler, P. M., \& Kano, Y. (1992). Can test statistics in covariance structure-analysis be trusted. Psychological Bulletin, 112, 351-362. doi:10.1037/0033-2909.112.2.351 
Keltner, D., \& Buswell, B. N. (1996). Evidence for the distinctness of embarrassment, shame, and guilt: A study of recalled antecedents and facial expressions of emotion. Cognition \& Emotion, 10, 155-171. doi:Doi 10.1080/026999396380312

Ketelaar, T., \& Au, W. T. (2003). The effects of feelings of guilt on the behaviour of uncooperative individuals in repeated social bargaining games: An affect-as-information interpretation of the role of emotion in social interaction. Cognition and Emotion, 17, 429-453. doi:10.1080/02699930143000662

Konecni, V. J. (1972). Some effects of guilt on compliance: A field replication. Journal of Personality and Social Psychology, 23, 30-32. doi:10.1037/h0032875

Landman, J. (1993). Regret: The persistence of the possible: New York: Oxford University Press.

Lazarus, R. S. (1991). Emotion and adaptation. New York: Oxford University Press.

Martinez, L. F., Zeelenberg, M., \& Rijsman, J. B. (2011). Regret, disappointment and the endowment effect. Journal of Economic Psychology, 32, 962-968. doi:DOI 10.1016/j.joep.2011.08.006

Mikulincer, M. (1988). Reactance and helplessness following exposure to unsolvable problems the effects of attributional style. Journal of Personality and Social Psychology, 54, 679686.

Mikulincer, M. (1994). Human learned helplessness : A coping perspective. New York: Plenum Press.

Moors, A. (2009). Theories of emotion causation: A review. Cognition and Emotion, 23, 625662. doi:10.1080/02699930802645739

Moors, A. (in press). Flavors of appraisal theories of emotion. Emotion Review.

Moors, A., \& Scherer, K. R. (Eds.). (2013). The role of appraisal in emotion: NY: Guilford Press.

Parkinson, B. (1997). Untangling the appraisal-emotion connection. Personality and Social Psychology Review, 1, 62-79.doi:10.1207/s15327957pspr0101_5

Parkinson, B., \& Illingworth, S. (2009). Guilt in response to blame from others. Cognition \& Emotion, 23, 1589-1614. doi:10.1080/02699930802591594

Parrott, W. G., \& Hertel, P. (1999). Research methods in cognition and emotion. In T. Dalgleish \& M. Power (Eds.), The handbook of cognition and emotion (pp. 61-81): Chichester: John Wiley \& Sons.

Rawlings, E. I. (1968). Witnessing harm to other: A reassessment of role of guilt in altruistic behavior. Journal of Personality and Social Psychology, 10, 377-380. doi:10.1037/H0026819

Reb, J., \& Connolly, T. (2009). Myopic regret avoidance: Feedback avoidance and learning in repeated decision making. Organizational Behavior and Human Decision Processes, 109, 182-189. doi:10.1016/j.obhdp.2009.05.002

Regan, D. T., Williams, M., \& Sparling, S. (1972). Voluntary expiation of guilt: A field experiment. Journal of Personality and Social Psychology, 24, 42-45. doi: $10.1037 / \mathrm{h} 0033553$

Regan, J. W. (1971). Guilt, perceived injustice, and altruistic behavior. Journal of Personality and Social Psychology, 18, 124-132. doi:10.1037/h0030712 
Roseman, I. J. (2001). A model of appraisal in the emotion system: Integrating theory, research, and applications. In K. R. Scherer, A. Schorr \& T. Johnstone (Eds.), Appraisal processes in emotion: Theory, methods, research (pp. 68-91): New York: Oxford University Press.

Roseman, I. J. (2011). Emotional behaviors, emotivational goals, emotion strategies: Multiple levels of organization integrate variable and consistent responses. Emotion Review, 3, 434-443. doi:Doi 10.1177/1754073911410744

Roseman, I. J., Antoniou, A. A., \& Jose, P. E. (1996). Appraisal determinants of emotions: Constructing a more accurate and comprehensive theory. Cognition and Emotion, 10, 241-277. doi:10.1080/026999396380240

Roseman, I. J., Wiest, C., \& Swartz, T. S. (1994). Phenomenology, behaviors, and goals differentiate discrete emotions. Journal of Personality and Social Psychology, 67, 206221. doi:10.1037//0022-3514.67.2.206

Rosseel, Y. (2012). Lavaan: An r package for structural equation modeling. Journal of Statistical Software, 48, 1-36.

Russell, J. A. (2009). Emotion, core affect, and psychological construction. Cognition \& Emotion, 23, 1259-1283. doi:10.1080/02699930902809375

Satorra, A. (2000). Scaled and adjusted restricted tests in multi-sample analysis of moment structures. In R. D. H. Heijmans, D. S. G. Pollock \& A. Satorra (Eds.), Innovations in multivariate statistical analysis. A festschrift for heinz neudecker (pp. 233-247). London: Kluwer Academic Publishers.

Satorra, A., \& Bentler, P. M. (1994). Corrections to test statistics and standard errors in covariance structure analysis. In A. von Eye \& C. C. Clogg (Eds.), Latent variables analysis:Applications for developmental research (pp. 399-419): Sage, Thousands Oaks, CA.

Satorra, A., \& Bentler, P. M. (2010). Ensuring positiveness of the scaled difference chi-square test statistic. Psychometrika, 75, 243-248. doi:10.1007/s11336-009-9135-y

Scherer, K. R. (1988). Criteria for emotion-antecedent appraisal: A review. In V. Hamilton, G. H. Bower \& N. H. Frijda (Eds.), Cognitive perspectives on emotion and motivation (pp. 89-126): Dordrecht, the Netherlands: Kluwer.

Scherer, K. R. (1994). Emotion serves to decouple stimulus and response. In P. Ekman \& R. J. Davidson (Eds.), The nature of emotion: Fundamental questions (pp. 127-130): New York/Oxford: Oxford University Press.

Scherer, K. R. (2005). What are emotions? And how can they be measured? Social Science Information Sur Les Sciences Sociales, 44, 695-729. doi:10.1177/0539018405058216

Scherer, K. R. (2009). The dynamic architecture of emotion: Evidence for the component process model. Cognition and Emotion, 23, 1307-1351. doi:10.1080/02699930902928969

Shani, Y., \& Zeelenberg, M. (2007). When and why do we want to know? How experienced regret promotes post-decision information search. Journal of Behavioral Decision Making, 20, 207-222. doi:10.1002/bdm.550

Shefrin, H., \& Statman, M. (1985). The disposition to sell winners too early and ride losers too long: Theory and evidence. The Journal of Finance, 40, 777-790. doi:10.2307/2327802

Sonnemans, J., \& Frijda, N. H. (1994). The structure of subjective emotional intensity. Cognition \& Emotion, 8, 329-350. doi:10.1080/02699939408408945 
Staw, B. M. (1976). Knee-deep in the big muddy: A study of escalating commitment to a chosen course of action. Organizational Behavior and Human Performance, 16, 27-44. doi:10.1016/0030-5073(76)90005-2

Struthers, C. W., Eaton, J., Shirvani, N., Georghiou, M., \& Edell, E. (2008). The effect of preemptive forgiveness and a transgressor's responsibility on shame, motivation to reconcile, and repentance. Basic and Applied Social Psychology, 30, 130-141. doi:10.1080/01973530802209178

Tangney, J. P., Miller, R. S., Flicker, L., \& Barlow, D. H. (1996). Are shame, guilt, and embarrassment distinct emotions? Journal of Personality and Social Psychology, 70, 1256-1269. doi:Doi 10.1037/0022-3514.70.6.1256

Tracy, J. L., \& Robins, R. W. (2006). Appraisal antecedents of shame and guilt: Support for a theoretical model. Personality and Social Psychology Bulletin, 32, 1339-1351. doi:Doi $10.1177 / 0146167206290212$

Tugade, M. M., Fredrickson, B. L., \& Barrett, L. F. (2004). Psychological resilience and positive emotional granularity: Examining the benefits of positive emotions on coping and health. Journal of Personality, 72, 1161-1190. doi:10.1111/j.1467-6494.2004.00294.x

van Dijk, W. W., van der Pligt, J., \& Zeelenberg, M. (1999). Effort invested in vain: The impact of effort on the intensity of disappointment and regret. Motivation and Emotion, 23, 203220. doi:10.1023/A:1021315314979

van Dijk, W. W., \& Zeelenberg, M. (2002). Investigating the appraisal patterns of regret and disappointment. Motivation and Emotion, 26, 321-331. doi:10.1023/A:1022823221146

Wortman, C. B., \& Brehm, J. W. (1975). Responses to uncontrollable outcomes: An integration of reactance theory and the learned helplessness model. In L. Berkowitz (Ed.), Advances in experimental social psychology (Vol. 8, pp. 277-336): New York: Academic Press.

Zeelenberg, M., \& Beattie, J. (1997). Consequences of regret aversion 2: Additional evidence for effects of feedback on decision making. Organizational Behavior and Human Decision Processes, 72, 63-78. doi:DOI 10.1006/obhd.1997.2730

Zeelenberg, M., Beattie, J., vanderPligt, J., \& deVries, N. K. (1996). Consequences of regret aversion: Effects of expected feedback on risky decision making. Organizational Behavior and Human Decision Processes, 65, 148-158. doi:DOI 10.1006/obhd.1996.0013

Zeelenberg, M., \& Breugelmans, S. M. (2008). The role of interpersonal harm in distinguishing regret from guilt. Emotion, 8, 589-596. doi:10.1037/a0012894

Zeelenberg, M., \& Pieters, R. (2004a). Beyond valence in customer dissatisfaction: A review and new findings on behavioral responses to regret and disappointment in failed services. Journal of Business Research, 57, 445-455. doi:Doi 10.1016/S0148-2963(02)00278-3

Zeelenberg, M., \& Pieters, R. (2004b). Consequences of regret aversion in real life: The case of the dutch postcode lottery. Organizational Behavior and Human Decision Processes, 93, 155-168. doi:DOI 10.1016/j.obhdp.2003.10.001

Zeelenberg, M., \& Pieters, R. (2006). Feeling is for doing: A pragmatic approach to the study of emotions in economic behavior. In D. De Cremer, M. Zeelenberg \& K. Murnighan (Eds.), Social psychology and economics: Mahwah, NJ: Erlbaum.

Zeelenberg, M., \& Pieters, R. (2007). A theory of regret regulation 1.0. Journal of Consumer Psychology, 17, 3-18. 
Zeelenberg, M., van Dijk, W. W., \& Manstead, A. S. R. (1998). Reconsidering the relation between regret and responsibility. Organizational Behavior and Human Decision Processes, 74, 254-272. doi:10.1006/obhd.1998.2780

Zeelenberg, M., van Dijk, W. W., Manstead, A. S. R., \& van der Pligt, J. (1998). The experience of regret and disappointment. Cognition and Emotion, 12, 221-230. doi:10.1080/026999398379727

Zeelenberg, M., van Dijk, W. W., Manstead, A. S. R., \& van der Pligt, J. (2000). On bad decisions and disconfirmed expectancies: The psychology of regret and disappointment. Cognition and Emotion, 14, 521-541. doi:10.1080/026999300402781

Zeelenberg, M., van Dijk, W. W., van der Pligt, J., Manstead, A. S. R., van Empelen, P., \& Reinderman, D. (1998). Emotional reactions to the outcomes of decisions: The role of counterfactual thought in the experience of regret and disappointment. Organizational Behavior and Human Decision Processes, 75, 117-141. 


\section{Footnotes}

${ }^{1}$ There were no effects of incentive for participation (money vs. course credit) on any of the dependent measures: the number of repair responses on self- or circumstances-agency trials, $t \mathrm{~s}<0.15, p \mathrm{~s}>.88$, the self-reported tendency to repair on self- or circumstances-agency trials, $t \mathrm{~s}$ $<0.95, p s>.35$, and feelings of regret and disappointment on self- or circumstances-agency trials, $t \mathrm{~s}<1.7, p \mathrm{~s}>.11$.

${ }^{2}$ In reality, there was no such algorithm. The program determined that $60 \%$ of the trials yielded an outcome of ten points.

${ }^{3} \mathrm{We}$ only analyzed trials on which repairing was impossible because only on those trials participants always had to decide themselves when to stop repairing. On trials on which repairing was possible, participants typically regained the ten points after a number of button presses and the fix button subsequently disappeared.

${ }^{4}$ Using other outlier criteria, no outlier criteria, or medians instead of means produced the same results. The same results were also observed when we analyzed the number of trials in which participants engaged in repair behavior $\left(M_{\text {self }}=95.79, S D_{\text {self }}=10.15 ; M_{\text {self }}=96.51, S D_{\text {self }}=\right.$ $7.14 ; t(25)=0.74, p=0.46)$, instead of the number of repair responses.

${ }^{5}$ All two tailed p-values.

${ }^{6}$ Participants also indicated the extent to which the event was a consequence of the behavior or choices of one or more other persons (other-agency) and a consequence of situational factors or circumstances (circumstances-agency). These items were not analyzed in the current study. 
${ }^{7}$ At this time, participants in the regret/disappointment condition rated feelings of disappointment/regret only because they already rated feelings of regret/disappointment at the beginning of the questionnaire.

${ }^{8}$ Sixty-three participants were disappointed in an outcome and 49 in a person. There were no significant differences between these groups with respect to the tendency to repair (tendency to change the situation, $t(110)<1, p=.39$, improve or rectify the situation, $t(110)<1, p=.96$, and undo the event, $t(110)=1.24, p=.16$. 
Table 1

Means (SDs), dependent sample t-tests, and effect sizes $\left(g_{a v}\right)$ for the manipulation check, the measures of the tendency to repair, and feelings for self-and circumstances-agency trials.

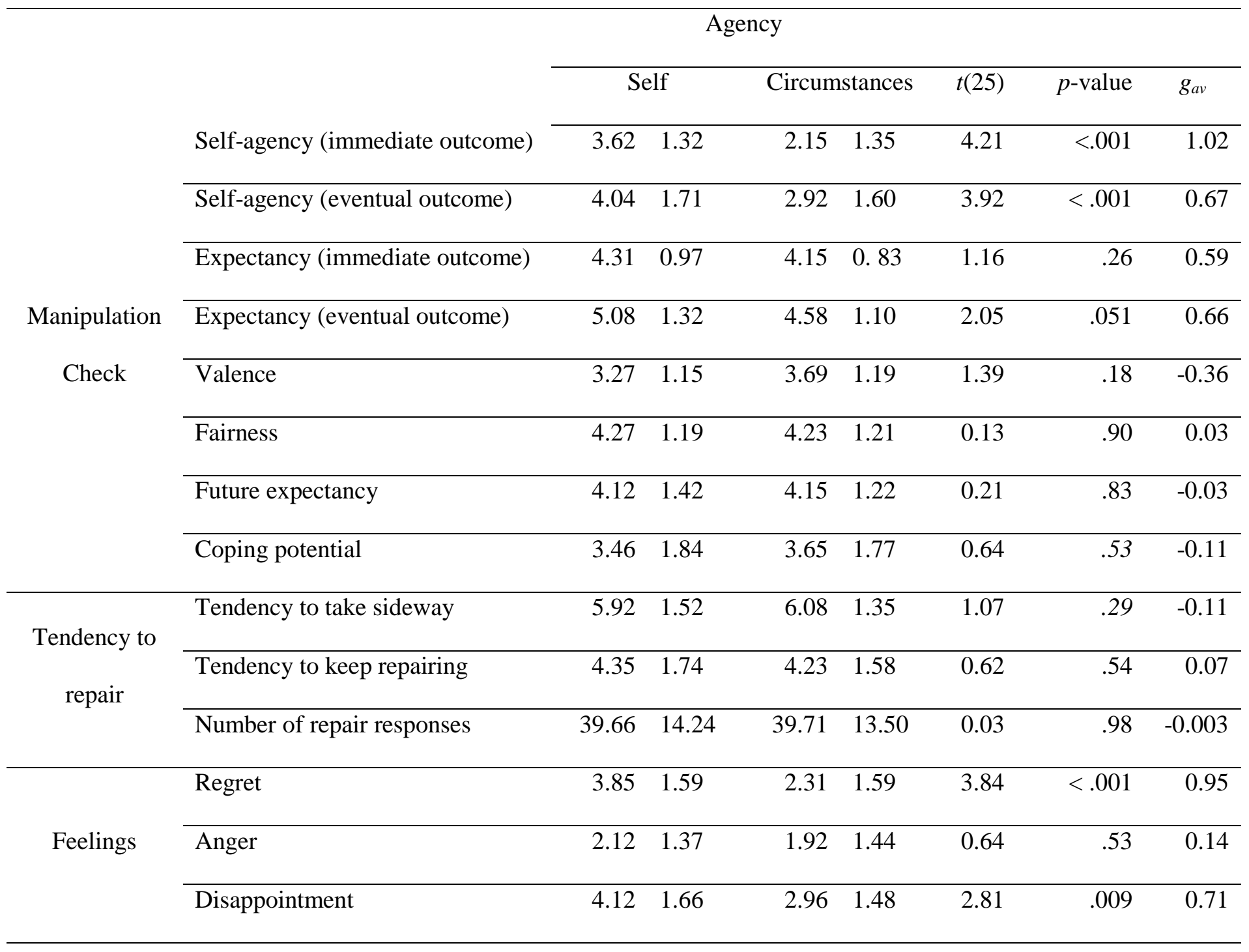


Table 2

Correlations between feelings and the tendency to repair for self-agency trials (Self), circumstances-agency trials (Circ), the difference score between self- and circumstances-agency trials (Diff), and the aggregated scores across self- and circumstances-agency trials (Total).

\begin{tabular}{|c|c|c|c|c|c|c|c|c|c|c|c|c|c|}
\hline & & \multicolumn{4}{|c|}{ Regret } & \multicolumn{4}{|c|}{ Disappointment } & \multicolumn{4}{|c|}{ Anger } \\
\hline \multirow{5}{*}{$\begin{array}{c}\text { Repair } \\
\text { behavior }\end{array}$} & & Self & Circ & Diff & Total & Self & Circ & Diff & Total & Self & Circ & Diff & Total \\
\hline & Self & $.01_{\mathrm{x}}$ & & & & $.39_{y}^{(*)}$ & & & & $.39_{\mathrm{x}}^{*}$ & & & \\
\hline & Circ & & $.16_{\mathrm{x}}$ & & & & $.39_{\mathrm{x}}^{(*)}$ & & & & $.29_{\mathrm{x}}$ & & \\
\hline & Diff & & & $.01_{\mathrm{x}}$ & & & & $.36_{\mathrm{x}}{ }^{(*)}$ & & & & $.01_{\mathrm{x}}$ & \\
\hline & Total & & & & $.12_{\mathrm{x}}$ & & & & $.45_{x}$ & & & & $.42_{\mathrm{x}}^{*}$ \\
\hline Self- & Self & $.22_{\mathrm{x}}$ & & & & $.50_{x}{ }^{*}$ & & & & $.14_{\mathrm{x}}$ & & & \\
\hline reported & Circ & & $.29_{\mathrm{x}}$ & & & & $.37_{\mathrm{x}}^{(*)}$ & & & & $.24_{\mathrm{x}}$ & & \\
\hline Repair & Diff & & & $.09_{\mathrm{x}}$ & & & & $.48_{\mathrm{y}}^{*}$ & & & & $.26_{\mathrm{x}}$ & \\
\hline Tendency & Total & & & & $.32_{\mathrm{x}}$ & & & & $.50_{\mathrm{x}}^{*}$ & & & & $.19_{\mathrm{x}}$ \\
\hline
\end{tabular}

${ }^{(*)} p<.10,{ }^{*} p<.05,{ }^{* *} p<.01$. Correlations for disappointment and anger received the subscript $\mathrm{x}$ if not different from the correlation for regret depicted on the same line $(p>.10)$, the subscript $x^{\prime}$ if marginally different from the correlation for regret depicted on the same line $(p<.10)$, and the subscript $y$ if significantly different from the correlation for regret depicted on the same line $(p<.05)$, . 
Table 3

Means (SDs), independent sample t-tests, and effect size (Hedges $\left.g_{s}\right)$ for the tendency to repair items, split up into those that include reference to self-agency (Confounded) and those that do not (Non-confounded).

\begin{tabular}{|c|c|c|c|c|c|c|}
\hline Item type & Item (short description) & Disappointment & Regret & $t$ & $p$ & $g_{s}$ \\
\hline \multirow{3}{*}{ Confounded } & Handle the situation differently & $5.91(2.61)$ & $6.80(2.65)$ & 2.54 & .012 & 0.34 \\
\hline & Want to turn back time & $6.26(2.95)$ & $7.94(1.83)$ & 5.15 & $<.001$ & 0.68 \\
\hline & Want a second chance & $6.61(2.68)$ & $7.31(2.37)$ & 2.08 & .039 & 0.26 \\
\hline \multirow{3}{*}{$\begin{array}{l}\text { Non- } \\
\text { onfounded }\end{array}$} & Change the situation & $6.10(2.67)$ & $5.63(2.53)$ & -1.35 & .18 & 0.18 \\
\hline & Improve or rectify the situation & $5.96(2.61)$ & $5.90(2.73)$ & -0.17 & .86 & 0.02 \\
\hline & Undo the event & $6.03(2.79)$ & $6.29(2.68)$ & 0.72 & .47 & 0.10 \\
\hline
\end{tabular}




\section{Figure Captions}

Figure 1 Hypotheses of Study 1 (H1, H2a, H3a) about the inter-relations between the appraisal of self-agency, the tendency to repair, and the feeling of regret.

Figure 2 Picture of the maze.

Figure 3 Pictures of the trials. Panel A depicts the starting phase of a self-agency trial (the word ME appears in front of the avatar); panel B depicts the starting phase of a circumstances-agency trial (a die appears in front of the avatar). Panel $\mathrm{C}$ depicts the outcome phase of a goalincongruent trial. Panel D depicts the outcome phase of a goal-congruent trial.

Figure 4 Hypotheses and results of the Structural Equation Model of the relations between the appraisal of self-agency, the appraisal of coping potential, the feeling of regret, the feeling of disappointment, and the tendency to repair. Full lines represent significant relations ${ }^{*} p<.05$; $* * p<.01 ; * * * x<.001)$; dashed lines represent non-significant relations $(p>.05)$. 
Figure 1.

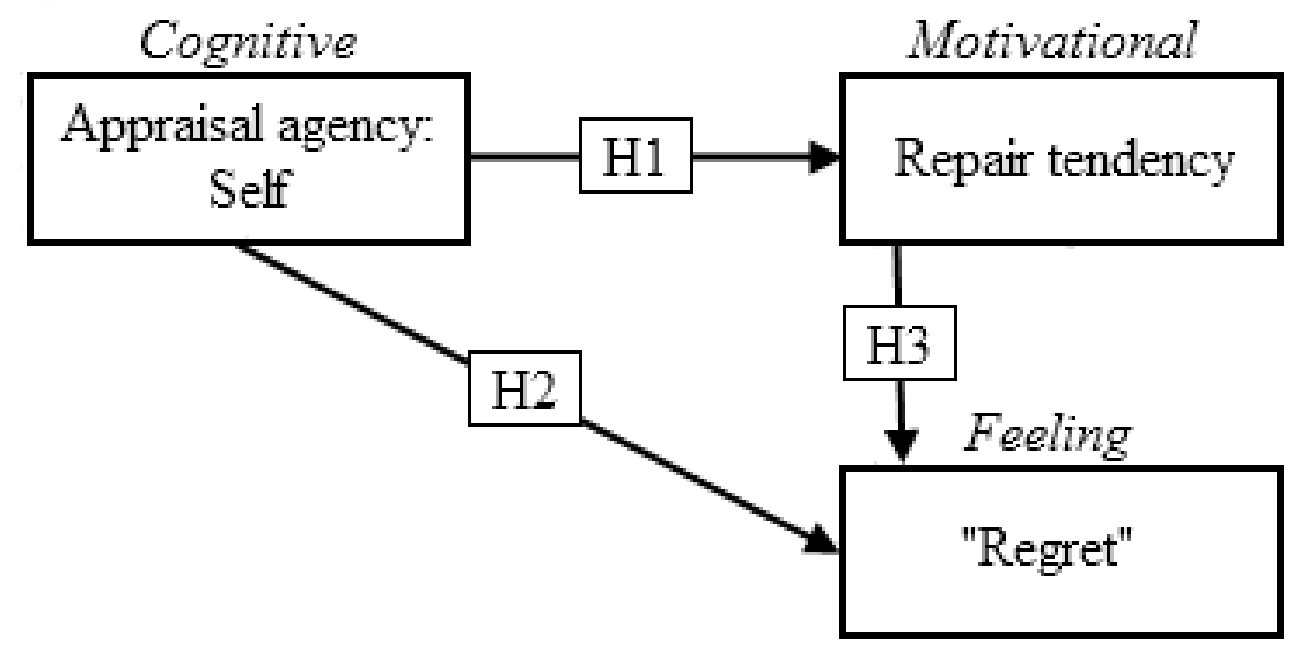


Figure 2.

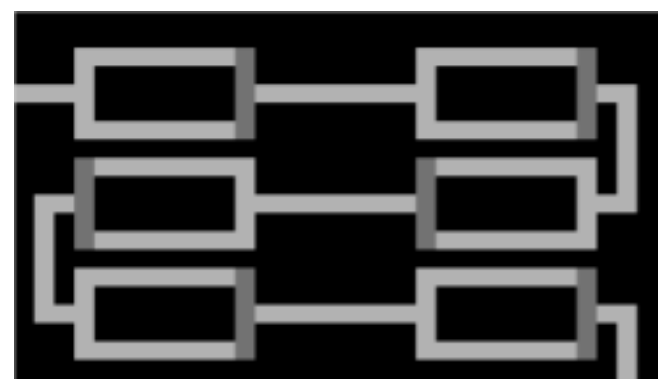


Figure 3.

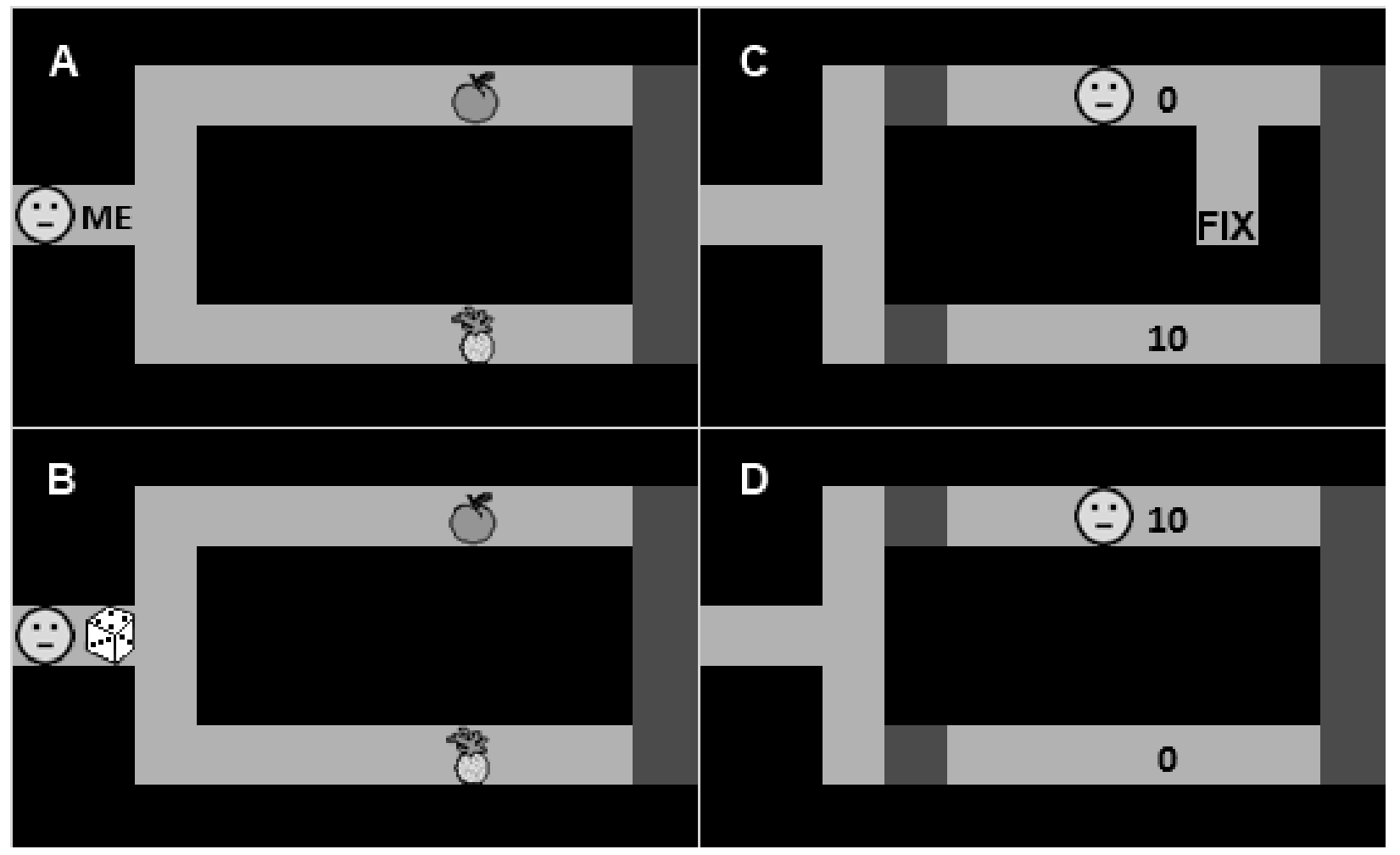


Figure 4.

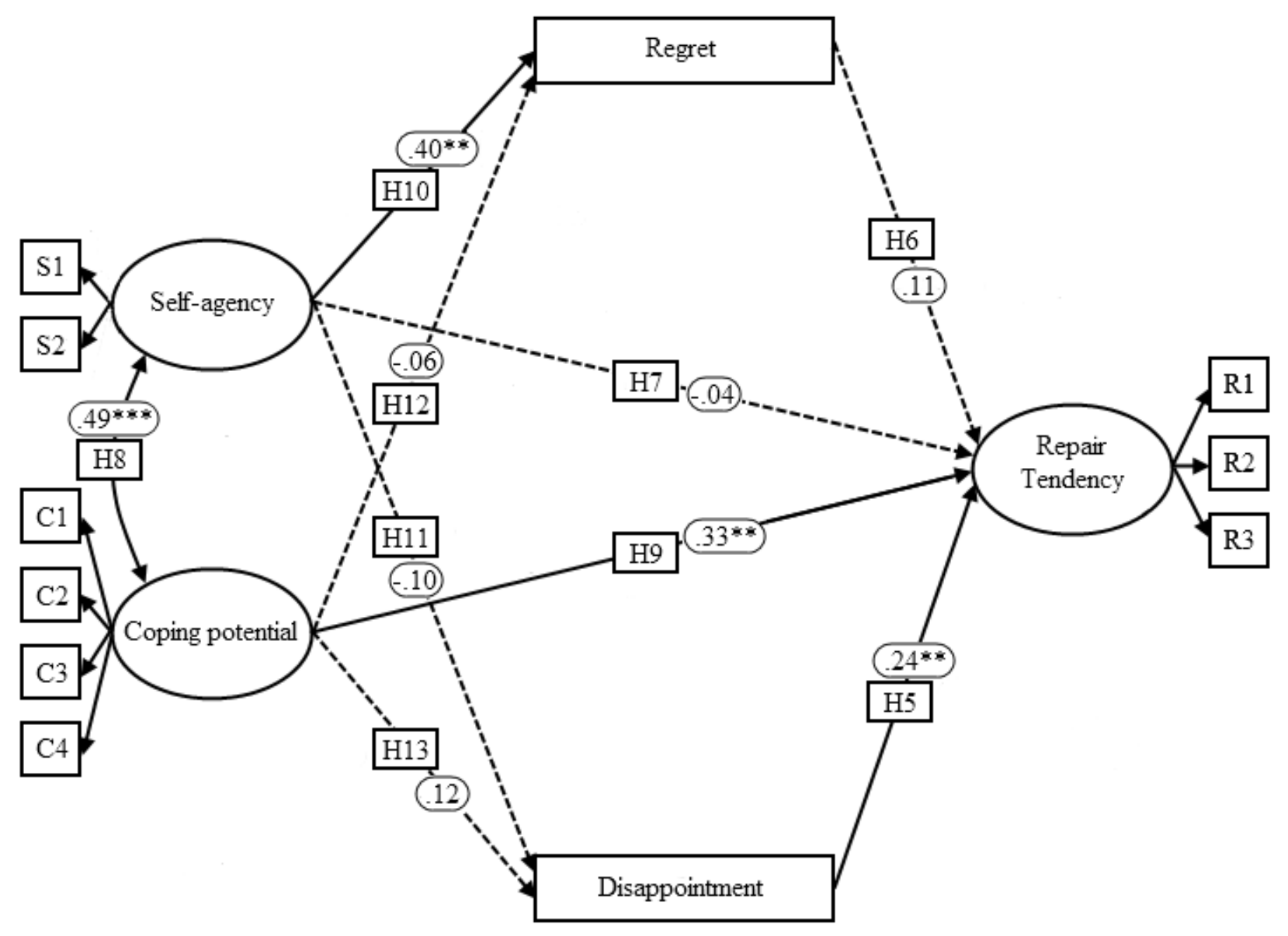

